\title{
Finding Co-solvers on Twitter, with a Little Help from Linked Data
}

\author{
Milan Stankovic ${ }^{1,3}$, Matthew Rowe ${ }^{2}$, and Philippe Laublet ${ }^{3}$ \\ ${ }^{1}$ Hypios Research, 187 rue du Temple, 75003 Paris, France \\ ${ }^{2}$ Knowledge Media Institute, The Open University, Milton Keynes, UK, MK7 6AA \\ ${ }^{3}$ STIH, Université Paris-Sorbonne, 28 rue Serpente, 75006 Paris, France \\ mail@milstan.net, m.c.rowe@open.ac.uk, \\ philippe. laubletaparis-sorbonne.fr
}

\begin{abstract}
In this paper we propose a method for suggesting potential collaborators for solving innovation challenges online, based on their competence, similarity of interests and social proximity with the user. We rely on Linked Data to derive a measure of semantic relatedness that we use to enrich both user profiles and innovation problems with additional relevant topics, thereby improving the performance of co-solver recommendation. We evaluate this approach against state of the art methods for query enrichment based on the distribution of topics in user profiles, and demonstrate its usefulness in recommending collaborators that are both complementary in competence and compatible with the user. Our experiments are grounded using data from the social networking service Twitter.com.
\end{abstract}

Keywords: Linked Data, Twitter, Collaborator Recommendation.

\section{Introduction}

Modern challenges that science and engineering worlds are facing today are often interdisciplinary and require research cooperation of teams of people in order to produce good solutions. Analysis of tendencies in research publications [1] shows that more and more multi-university teams produce accepted papers. Similarly, industrial innovation challenges often require a collaborative effort of experts from across different disciplines to work together. In this sense, innovation problem solving platforms, such as Innocentive ${ }^{1}$, have started to propose problem challenges for teams of problem solvers. Supporting users in the task of forming productive multidisciplinary teams therefore plays an important role in a multitude of innovation-related situations.

Existing social studies [2] on the topic of forming teams investigate people's preferences when it comes to the choice of co-workers, they underline the importance of co-worker similarity (both in terms of shared interests/expertise and in terms of shared social connections) together with the expertise of co-workers given the work task. Conveniently, more and more data about users (i.e. their social connections, topics of interest, their work) is available on the Web, this opens the way for a

${ }^{1}$ http: / / www. innocentive.com/ 
co-worker recommendation approach that takes into account those different qualities of the user and performs useful recommendations.

In this paper we are addressing the challenge of recommending potential co-solvers to people facing an innovation or research problem for which they are only partially competent. This recommendation is performed based on the data available in social networks (both the user's social graph and the user generated content), by taking into account both the compatibility of candidates with the user, and the complementarity of their competence. The research questions driving our work are: how can we suggest appropriate co-solvers, which were potentially, previously unknown to the user? And: what information can be used to enrich initially available user and problem data to provide the best suggestions? In exploring these two research questions we have devised an approach to recommend co-solvers for open innovation problems. The contributions from this work are three-fold, and are as follows: (1) Profile Expansion: we present methods for expanding topics that measure semantic relatedness between topics using the linked data graph; (2) Similarity Measures: we describe three similarity measures that exploit either relations between topic concepts or social connections; (3) Evaluation: we assess the performance of our approach when different profile expansion and similarity measures are used through two user studies.

The remainder of the paper is organized as follows: In section 2 we describe the Open Innovation related scenario of use for which we developed our approach and we present related work covering a) the recommendation of co-workers; b) expert finding, and; c) measures of semantic relatedness. Our core approach is presented in section 3 together with different alternatives for several parts of the recommendation process. In section 4 we present two user study-based evaluations of our approach executed over the social network Twitter.com. In section 5 we present our directions of future work and conclude the paper.

\section{Background and Related Work}

\subsection{Open Innovation and the Need for Co-solvers}

Open Innovation (OI) [3] has emerged as way to accelerate industrial innovation by looking for diverse and unexpected perspectives to innovation problems outside of the company. OI platforms, such as hypios.com, Innocentive.com, and NineSigma.com have emerged to support this practice. Those platforms allow innovation seekers to post their problems for solving by Web users and award the best solutions. Such innovation platforms aim to allow anyone who has some knowledge or idea to participate in the innovation process. They value diversity in solutions over the acclaim and level of expertise of solvers, as studies have shown that people with marginal competence in the problem's domain, but experts in some other domain, are able to bring more innovative solutions [4], often by transposing ideas from one field to another. Our work on co-solver recommendation, although generalisable to any use case of finding collaborators, is principally oriented at finding people to work with on the Open Innovation problems. This implies a preference towards candidates who are able to bring a novel perspective to solving the problem over those who are acclaimed experts in the exact domain of the problem. 


\subsection{Recommending People}

A number of approaches have been proposed for challenges similar to ours, most notably for recommending people to befriend in social networks. Those recommender systems usually rely on a measure that quantifies the similarity of two users or the similarity of a user profile with a given document or query. Spertus et al. [5] present an empirical comparison of six measures of similarity for recommending communities to members of the Orkut social network. They found the cosine similarity measure to show the best empirical results against other measures. In [6] a transitive notion of user similarity is proposed to address the scarcity of user profiles in collaborative filtering systems when recommending movies. [7] relies only on Jacquard's similarity to compare different sources from which user similarity may be mined (friendships, interests, activities in same places, etc.) in terms of their performance in different scenarios. Another study, presented in [8] shows that algorithms based on a user's social connections are better in recommending known contacts, while those based on the similarity of user-generated content perform better at suggesting new contacts. Those studies however do not provide implications for the use case of recommending co-workers. In addition to similarity, some systems like Facebook, consider affinity towards a user calculated based on the level of mutual interactions on Facebook ${ }^{2}$.

A number of multi-criteria recommender systems have been proposed, mostly using a (linear) combination of different similarity functions to deliver recommendations. For instance a collaborative filtering system for recommending similar users [9] uses a machine learning approach to learn weight factors of different utility functions. [10] also uses a multitude of criteria derived from different interactions users make with Web objects. [11] develops a machine learning approach for leveraging content and social criteria in providing folksonomy-based recommendations, especially on Flickr. However, to our best knowledge such approaches have not been used to suggest co-solvers.

\subsection{Expert Finding}

A multitude of approaches for expert finding from a range of sources like blogs, scientific literature, corporate social networks, etc. have been proposed in the past many of which in TREC conferences [12]. Many of such approaches exploit microposts, including tweets, for expert profiling, thereby confirming the viability of this source for co-solvers. For instance, Zoltan and Johan [13] propose a system for the extraction of ontological topic concepts from tweets, topics are then weighted by their importance to the expert profile. Analysis of persistency of topics [14] as well as the awareness of profile dynamics $[15,16]$ have been shown to improve user profiling, over static approaches. Twitter lists have been shown to be a rich source for user profiling [17]. The construction of expert profiles from Twitter data using topic models is also proposed by Wagner [18].

Most of these existing approaches focus on finding people with expertise in a particular topic, while the question of fitting an expert to an innovation task involving

${ }^{2}$ http: / / techcrunch.com/2010/04/22/facebook-edgerank/ 
a multitude of topics has not yet been fully explored. To the best of our knowledge, existing approaches do not respond to the needs of OI scenarios, where the requirements in terms of expertise of a potential problem solver are slightly different than those used to select experts in most expert-finding approaches. The necessary focus on getting diverse and laterally relevant experts has, to the best of our knowledge, also not been the focus of the existing expert-finding approaches.

\subsection{Measures of Semantic Relatedness Usable for Profile Expansion}

Our approach for co-solver recommendation exploits the semantic relatedness of individuals through the linked data graph based on their topical expertise and interests. Measures of semantic relatedness (MSRs) have been proposed for use in a variety of tasks and can be split into two major categories: 1) graph-based measures and; 2) distributional measures.

Graph-based measures make use of semantic (e.g., hyponymy or meronymy) and/or lexical (e.g., synonyms) relationships within a network (graph) of concepts to determine semantic proximity between the concepts. For example, [19] exploits the hypernym graphs of Wordnet ${ }^{3}$; whereas [20] use the ODP taxonomy ${ }^{4}$ and [21] relies on the graph of Wikipedia categories to provide recommendations. Among graph measures used to calculate the semantic proximity of concepts shortest path is one of the most common, and is often enhanced by taking into account the informational content of nodes in the graph [22]. To the best of our knowledge these approaches have not been applied to knowledge bases of comparable size and richness to that of DBPedia $^{5}$. Even the Wikipedia-based measures that we found only used information about categories and did not leverage other information present in DBPedia. Our own MSR, hyProximity that we will describe shortly, builds upon the existing graph-based measures but is highly adapted to the rich structure of Linked Data sources, as it leverages different types of relations that concepts may have in the graph.

Distributional measures mostly explain semantic relatedness through common use, usually by leveraging co-occurrence of concepts, and mostly do not make connections over the meaning of terms or concepts. For example, the approach presented in [23] uses co-occurrence in text of research papers to establish a notion of word proximity, while others rely on other sources such as search results [24]. In addition to the measures of semantic relatedness, some authors propose the use of pseudo relevancefeedback to enrich an initial set of topics in a query, most notably in the domain of expert search. In particular, pseudo relevance-feedback is used to enlarge expert search queries based on additional keywords appearing in a number of top ranked documents [25] or top ranked user-profiles [25, 26]. The diversity of topics in expert profiles has been shown to negatively impact the quality of results when more narrow results are sought. No evaluation has been provided for the case when broadening of the space of found experts is desired for OI purposes.

\footnotetext{
${ }^{3}$ http: / / wordnet.princeton.edu/

${ }^{4}$ http : / / www. dmoz.org/

${ }^{5}$ While DBPedia contains more then 3.5 million concepts, the current version of Wordnet has 206941 word-sense pairs, and ODP has half a million categories.
} 


\section{Recommending Co-solvers}

Our general approach for recommending co-solvers is based on a user's social connections and/or the content he/she created. The general process is applicable on any social network with user-generated content, however we discuss our concrete implementation of the process on data from the social network Twitter.

\subsection{General Approach}

In our general approach, a Web user (called a seed user) approaches our system with the intention to find potential collaborators for a particular research challenge or innovation problem (called problem hereafter). He provides the text of the problem/challenge and gives some identifier that he uses on a social networking system, this allows access to: (1) his social connections and (2) some content that he created. The system then proceeds with the creation of profiles for both the user and the problem. Those profiles contain Linked Data identifiers of topics extracted from the provided textual elements (from the text of the problem/challenge in the case of the Problem Profile or from the content that the user has created in the case of the User Profile). Optionally, an additional phase of profile enrichment may be performed (called Profile Expansion). This functions by expanding the initial profiles in order to broaden their topics and thus compensate for any incompleteness. - i.e. where the topics may be too specific. Similarity scoring is performed over a base of candidate user profiles in order to select those candidate users that: (1) are the most similar to the seed user and; (2) whose profile fits the given innovation problem. Similarity scoring can work both with the initial user and problem profile as well as with the extended ones. Particular similarity functions will be further discussed in Section 3.3.

\subsection{Profiling}

In the profiling phase, user and problem profiles are created from the provided textual elements (posts and biography in the case of user profiles and problem text in the case of problem profiles). The topic profiles (denoted TP in equation (1)), regardless of the type of entity (user or problem) that they concern, are sets of tuples assembled from a Linked Data URI of a topic concept and a value $w$ representing the importance of this particular topic for the profile. In essence, this topic profile is a concept vector, where each element's index represents a unique concept. The value stored in a given element is the frequency of the concept's appearance in either: a) the user's past information or; b) the problem definition. In the phase of profile expansion, the values $w$ of additional related topics correspond to the relatedness of the topic to the given profile, as expressed by the particular measure of semantic relatedness used to perform the profile expansion.

$$
T P(\text { entity })=\left\{\left(U R L_{1}, w_{1}\right), \ldots,\left(U R I_{n}, w_{n}\right)\right\} \quad(1) \quad S P(\text { user })=\left\{\text { user }_{1}, \ldots, \text { user }_{n}\right\}
$$


Different operations are possible over the topic profiles. For instance, in our work we will rely on the difference $\mathrm{TP}$ (problem)-TP(user), called difference topics, that represents the topics found in the problem topic profile that are not found in the topic profile of the seed user - this derives the topics for which there is no record of seed user's past knowledge. In addition to topic profiles, social profiles (denoted SP in equation (2)) are also created for the users, and they contain the list of user's social connections:

\subsubsection{Extraction of Topics}

Extraction of topics from text plays a crucial role in the profiling phase. When we refer to topics in our work, we consider concepts of general human knowledge that have unique identifiers - e.g. a URI - and are described as resources somewhere in the Linked Data cloud. Descriptions of such concepts may be found, for instance, in knowledge bases such as Freebase.org or DBpedia.org. A number of entity recognition services propose the extraction of such concepts from given text, such as Zemanta $^{6}$, OpenCalais ${ }^{7}$, DBpedia Spotlight ${ }^{8}$ etc. In our experiments we use Zemanta for topic extraction. This service has been shown to perform well (among the best) for the task of recognizing Linked Data entities from text in recent evaluations [27].

\subsubsection{User Profiling on Twitter}

In our particular implementation on Twitter, we use the user's biography and a number of last tweets (300 used in particular experiments) to extract the topics. The DBpedia URIs of extracted topics are stored in TP along with the frequency of their occurrence. This approach assures that the most recent interest topics are taken into account. On the other hand it is not very restrictive, as any topic tweeted about may be considered as part of a user's profile. Our approach favours the broader view of topics in accordance with the need for laterality and inclusion of people with borderline interest/expertise in a particular topic, who might bring innovative perspectives to the problem solving process - essential for our Open Innovation scenario. In cases where it is necessary to assure that only topics for which the user is really experienced about are represented, it is possible to use a more restrictive approach such as one of those proposed in [14], [15], and [16], mostly making use of the dynamics and persistence of topics in user's tweets.

\subsubsection{Creating Candidate User Profiles}

Ideally, candidate users should be all the users of the Web. While it is theoretically possible to construct such a base of user profiles, most real world systems are confronted with constraints in terms of both access to user data and processing time, and thus have to restrict themselves to a set of candidate user profiles, most likely to be selected by the recommender system. In our case, for each seed user and problem, we perform searches on the raw social network data. In particular we perform two types of search: (1) to find all the users in the social proximity of the seed user, i.e. friends of friends, and (2) to find all the users corresponding to the topics found in the problem

\footnotetext{
${ }^{6}$ http: / / developer. zemanta.com

${ }^{7}$ http: / / www. opencalais.com/

${ }^{8}$ http: / / dbpedia. org/spotlight
} 
and the seed user profile, as found by the search function of the particular social network used - in the case of Twitter using their built-in search functionality. Users found by those different queries constitute a base of candidate user profiles for every recommendation. These two particular ways of harvesting candidate users correspond to the general intention of finding people similar to the seed user (in the sense of interests and social connections) and relevant for the topics of the problem. Users that our seed user are already friends with are eliminated from the possible recommendations as we assume that they are known to the seed user and as such would not represent valuable discoveries. In cases where they are considered relevant, it is relatively easy to tweak the system to also include the user's friends in the recommendations.

\subsection{Core Similarity Measures and Similarity Scoring}

In accordance to our criteria described in the introduction, the ranking of the candidate users should favour the candidates: whose profile topics are similar to the topics in the seed user's profile (interest similarity); whose social connections are similar to the social connections of the seed user (social similarity) and whose profile topics are similar to the difference topics (similarity with the difference topics). In order to enforce such a ranking we use measures of similarity that enable the measurement of each of the abovementioned aspects. We also use a combined measure in order to favour candidates who satisfy all of the above criteria. All the similarity measures operate over TP and SP vectors defined in \$3.2. As these measures take vectors as input we can apply two well established functions for vector similarity Weighted Overlap and Cosine Similarity:

- Weighted Overlap represents the sum of weights in the seed user's profile, for all topics that the seed and candidate user profiles have in common. The use of this simple measure can be observed in many approaches, like [28].

- Cosine similarity measure is another commonly used vector similarity measure $[29,30]$ and it is defined as the cosine of the angle of the two vectors to be compared.

We define three measures of similarity, each of which are used with the above functions:

- Interest Similarity - The similarity of topic profiles TP of the seed user and a candidate user. When applied in this way we will refer to our functions with a suffix $t$, e.g., WeightedOverlap ${ }_{t}$, Cosine $_{t}$;

- Social Similarity - The similarity of social connection profiles SP of the seed user and the candidate user. When used in this way, we will refer to our functions with a suffix s, e.g., WeightedOverlap ${ }_{\mathrm{s}}$, Cosine $_{\mathrm{s}}$;

- Similarity with difference topics - The similarity between the topic profile TP of a candidate user and the vector of difference topics. When used in this way, we will refer to our functions with a suffix $d t$, e.g., WeightedOverlap ${ }_{d t}$, Cosine $_{d t}$.

In order to aggregate the values of all 3 different similarity measures we use a composite similarity measure. Given that the values of the elementary similarity measures are in the range $[0,1]$, and that they are normalized to this range, we use the 
product $(\mathrm{PC})$ of the three elementary measures as the aggregate measure. For instance $\operatorname{PC}\left(\right.$ Cosine $\left._{\mathrm{t}}, \operatorname{Cosine}_{\mathrm{s}}, \operatorname{Cosine}_{\mathrm{dt}}\right)=\operatorname{Cosine}_{\mathrm{t}} \cdot \operatorname{Cosine}_{\mathrm{s}} \cdot \operatorname{Cosine}_{\mathrm{dt}}$. Alternatively it is possible to use the sum of weighted values of elementary similarity measures, in which case the weights may be adjusted by a machine-learning approach [9] in order to adapt to the preference of each user. The PC measure, as opposed to any linear combination of elementary functions, penalizes the candidates that rank extremely poorly at any single similarity function $(0 \bullet x=0)$, regardless of the high ranking at another function. The candidates ranked highly at only one similarity function could therefore not be ranked better then those being similar in all required aspects.

\subsection{Profile Expansion Using Semantic Relatedness Measures}

Prior to calculation of similarity measures it is possible to enrich the seed user profile as well as the problem profile with additional topics that are related to the topics initially found in their topic vectors. Figure 2 shows a graph through which the seed user and the problem are linked to the potential candidate co-solvers. While the topics found initially in the seed user profile (T1 and T2) and in the problem profile (topics T1, T3, T4 and T5) do lead to some potential co-solvers, it is possible to consider the semantic relatedness of topic concepts and in that way reach a larger set of candidate users and in general have a richer view of the similarity of profiles. By semantic enrichment of profiles we consider adding topics to an initial profile that are found to be related to the initial profile's topics according to a particular notion of semantic relatedness. This notion of semantic relatedness of topics may be derived in different ways. In this section we present 3 of such different measures: a) distributional semantic relatedness measure; b) hyProximity - a Linked Data-based measure of semantic relatedness - and; c) pseudo relevance-feedback.

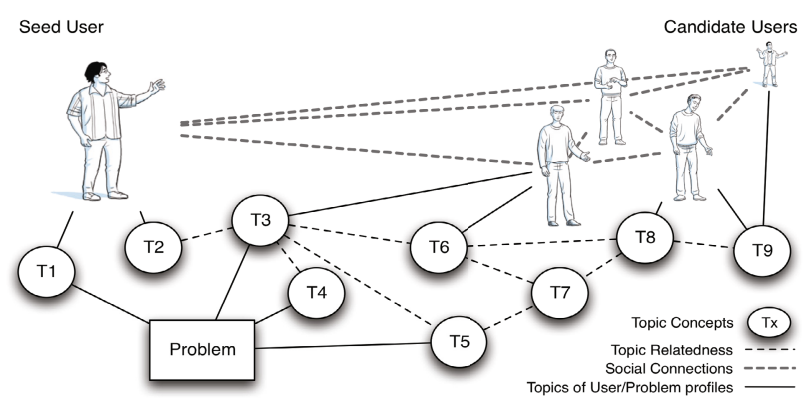

Fig. 1. The Graph of Social and Semantic Connections

The first step in profile enrichment is to generate relevant concepts (according to the measure used) for each topic present in the initial profile. This expansion is performed by first identifying related concepts to the original profile topics - e.g. T3 in Figure 1 when considering the seed user profile. The relatedness is then calculated between each profile topic and related concept - e.g. T2 with T3, and T1 with T3 - to derive individual relatedness values. The total relatedness between the profile and a given related concept is then given by the sum of these individual calculations, where 
the greater the cumulative relatedness the greater the relatedness between the profile and the related concept. The top-n topics are selected based on their cumulative relatedness - we set $n=30$ for our experiments.

\subsubsection{Distributional Measure of Semantic Relatedness (DMSR)}

$$
\operatorname{DMSR}_{\tau}\left(t_{1}, t_{2}\right)=\frac{\operatorname{ocurrence}\left(t_{1}, t_{2}\right)}{\operatorname{ocurrence}\left(t_{1}\right)+\operatorname{ocurrence}\left(t_{2}\right)}
$$

The distributional measure of semantic relatedness (DMSR) relies on co-occurrence of topics in profiles to conclude that two topics are similar. It is inspired from the distributional measures used in recommending keywords for search query term suggestion [24]. DMSR, expressed with the formula (3) considers two topics to be similar if they co-occur often in profiles. It represents the ratio of the number of joint occurrences of two topics $t_{1}$ and $t_{2}$ in user profiles and the sum of their individual occurrences. It is calculated over $\tau$ - the set of all user profiles taken into account as potential user candidates.

\subsection{2 hyProximity (HPSR)}

HyProximity is a measure of semantic relatedness (HPSR) that relies on Linked Data graphs of topic concepts. We have developed this notion for the purposes of discovering laterally relevant topics for solver finding purposes. While only a short summary of the notion is presented here, our previous paper [31] provides more detailed descriptions together with corresponding algorithms and evaluations.

In data sets rich with topics of general human knowledge, such as DBPedia and Freebase, concepts are usually related using properties that might be classified in two main types:

- Hierarchical links - Those created over properties that help organize the concepts in classes (e.g., rdf:type ${ }^{9}$ and rdfs:subclassOf) or categories (e.g., dcterms:subject and skos:broader). The links created by those properties connect a concept to a category - the one serving to organize other concepts into classes.

- Transversal links - Those created between ordinary, non-category concepts over properties that connect concepts without the aim to establish a classification or hierarchy. Those properties might create explicit links (connecting two concepts directly) or implicit links (when two concepts have the same value for the same property). Through the analysis of concepts appearing in past open innovation problems and their solutions received on hypios.com, we have discovered that only a small set of properties participate in forming all of the links between concepts observed in OI scenarios. This set of properties, referred to as $\mathrm{P}$, consists of dbo:product, dbo:industry, dbo:service, dbo:product and dbp:products.

$$
\operatorname{HPSR}\left(t_{1}, t_{2}\right)=\sum_{C_{i} \in C\left(t_{1}, t_{2}\right)} i c\left(C_{i}\right)+\sum_{p \in P} \operatorname{link}\left(p, t_{1}, t_{2}\right) \cdot \operatorname{pond}\left(p, t_{1}\right)
$$

Our approach uses the links created over these two types of properties in different ways, appropriate to the nature of those links. According to the equation (4) the value

\footnotetext{
${ }^{9}$ All the prefixes used in this paper can be looked up at http://prefix.CC
} 
of our HPSR measure for two topics $t_{1}$ and $t_{2}$ is the sum of valorisations of the connections achieved over hierarchical links (first component of the formula) and those achieved over transversal links (second component of the formula). In the treatment of hierarchical links we take all the common categories $C\left(t_{1}, t_{2}\right)$ of the two topics, and then for each common category $C_{i}$ we count the informational content [22] of this category as $-\log (p b)$ where $p b$ is the probability of finding the category in the graph of DBPedia categories when going from the bottom up. The sum of values for all common categories represents the strength of links established between $t_{1}$ and $t_{2}$ over the hierarchical properties. The transversal links are treated slightly differently. For each property $p$, from the previously defined set of relevant properties $\mathrm{P}$, we count the number of links connecting $t_{1}$ and $t_{2}$ over the property $p$ (given by function $\left.\operatorname{link}\left(p, t_{l}, t_{2}\right)\right)$ and weight them using weighting function $\operatorname{pond}\left(p, t_{1}\right)$. The value of the weighting function is calculated as $-\log (n / M)$, where $n$ is the number of other concepts to which the concept $t_{1}$ is connected over the same property $p$; and $M$ is the large constant larger then any possible value of $n$ (in our case it is the total number of concepts in DbPedia).

As our formula is not symmetric, i.e., $\operatorname{HPSR}(a, b)$ is not equal to $\operatorname{HPSR}(b, a)$, it is always calculated by putting the topics that belong to the seed user or to the difference topics as the first parameter, and the topics from the candidate user profiles as the second parameter.

\subsubsection{Pseudo Relevance-Feedback (PRF)}

Pseudo relevance-feedback (PRF) is a technique used to enlarge search queries, in which a number of best-ranked profile suggestions/results from a search or recommendation system is taken and the co-occurrence analysis is performed on them to discover the topics that appear frequently in the results, there are then used to enlarge the initial topics and re-run the recommendation process. We mentioned some of the examples of the use of pseudo relevance-feedback in the Background section. Computation wise, the measure of relatedness based on pseudo relevance feedback (denoted PRF) is calculated by the same formula (3) as DMSR with the exception that only a number (10 in our case) of the initially best ranked candidates are used as $\tau$ instead of considering the whole set of potential candidates.

\section{Evaluation}

In order to evaluate the performance of different similarity measures and approaches to profile expansion when providing co-solver recommendations we performed two experiments involving Twitter users. Recent studies show the growth of scholarly Twitter accounts ${ }^{10}$ and its use in communication in scientific communities, especially the Computer Science research community [32], thus making Twitter resourceful for co-solvers recommendations. We first created three multidisciplinary innovation problems ${ }^{11}$, inspired from descriptions of existing research challenges and projects that we found online, each involving the topics related to Semantic Web and to at least one

\footnotetext{
${ }^{10}$ Third of all scholars are said to have a Twitter account today http: / /www.scribd.com/doc/37621209/2010-Twitter-Survey-Report

${ }^{11}$ Available on our website http: / / research.hypios.com/?page_id=184
} 
other domain (in particular: Sensors, Online Advertising and Venture Capital Investments). We then used different alternatives of our method to suggest possible collaborators corresponding to our raters, by relying on candidate user profiles created according to our approach described in 3.2.2 and 3.2.3. In the first experiment $(\$ 4.1)$ we used a gold standard obtained from 3 raters and then assessed the performance of different permutations of profile expansion methods with the interest similarity measure and the difference topic measure. We omitted social similarity from this stage due to the differences in the social networks of the 3 solvers - as each solver has different potential candidates - the gold standard in this case uses the intersection of candidates recommended to each solver. In the second experiment (\$4.2) we evaluated all profile expansion methods with all ranking methods using a group of 12 raters. In this case we did not take interrater agreement for the gold standard, but instead evaluated performance on an individual basis. We were therefore able to include social similarity as a ranking technique and evaluate its performance. Performing these two studies allows the comparison between performance of different profile expansion and similarity measures when a) recommending co-solvers to a group of users - in the case of experiment 1 , and; b) recommending co-solvers to individual users in experiment 2 . To gauge the performance of different permutations of profile expansion and similarity measures we used the following evaluation metrics:

Discounted Cumulative Gain. (DCG) quantifies the value of items in the top- $n$ ranked suggestions as well as the quality of their ranking. For each ranking resulting from a particular ranking alternative, we take the 10 best-ranked user candidates and look at the ratings users generated for them. If the user candidate found at position $i$ is rated positively by users we take rating to be 1 , otherwise we consider it being equal to 0 . The importance of positively ranked candidates found on lower positions in a particular ranking is downgraded logarithmically in order to favour ranking alternatives that put the best candidates towards the top.

Average Precision. $\left(\mathrm{AveP}_{\mathrm{n}}\right)$ computes the average value of precision as a function of recall, on the interval recall $\in[0,1]$. For each position in a concrete ranking we calculate the precision up to that point, and the gain in recall acheived at that point as opposed to the previous point. The product of those gives an idea of the value a user would gain by looking at the suggestions up to a particular rank.

$$
D C G=\text { rating }_{1}+\sum_{i=2}^{10} \frac{\text { rating }_{i}}{\log _{2} i} \quad \text { (5) } \text { Ave }_{n}=\sum_{i=1}^{n} \text { precision }(i) \cdot \operatorname{\Delta recall}(i)
$$

\subsection{Evaluation 1}

In our first evaluation, we approached a group of 3 researchers from the field of the Semantic Web and presented them with our 3 problems for which they were collectively, as a group, only partially competent. For each rater we generated cosolver suggestions using different combinations of similarity measures and profile expansion approaches. We then took the top-10 suggestions from each different 
method and mixed all the suggestions together in one randomized list. This resulted in reasonably sized lists (i.e., 30-50), as some users were recommended by several methods, but on the other hand limited the possibilities of evaluation to the methods defined prior to user rating. The raters then rated candidates by answering if they would work with the suggested user on the given innovation problem. Raters were instructed to base their ratings on a holistic perception of suitability of the suggested user, and only positively rate the users who are both competent and who seem to be potential co-workers. Prior to calculation of any performance measures we calculated the inter-rater agreement using the kappa statistic defined in [33] for each pair of raters. The value of $k$ was, at first, inferior to the threshold of 0.6 for some of the rater pairs. We then allowed the raters to anonymously see the ratings of other group members and alter their ratings if they felt the others were right. After the second round the interrater agreement was superior to 0.6 for all 3 problems and for all problems $(0.74$ on average). We then used the majority agreement between raters to derive our decision labels for each candidate user (i.e., recommended or not recommended).
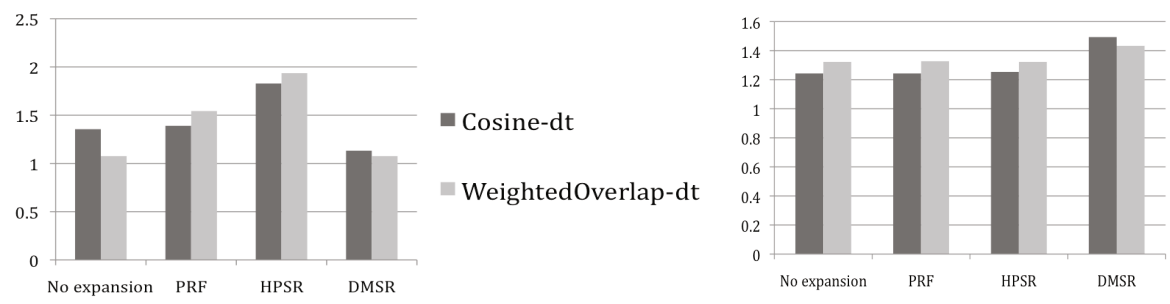

Fig. 2. DCG of rankings obtained with different methods of expansion applied to problem profiles (right) and to seed user profiles (left)

DCG values for similarity measures based on Cosine and Weighted Overlap functions run with all three expansions methods are shown on Figure 2. In the case of rankings based on the similarity with the difference topics, it is clear that the HSPR method of profile enrichment dominates the other expansion methods. This method is much less efficient when it comes to ranking based on the interest similarity of candidate users to the seed users, where DMSR slightly outperforms the other methods, with a little improvement over the standard approach. The figure shows average values over all 3 problems, and the differences in method performance have been confirmed to be significant by the $\mathrm{T}$ test $(\mathrm{p}<0.05)$ for HPSR with DMSR in similarity with difference topics, but not in the case of interest similarity. It should be noted that our expansion methods are not applicable to the calculation of social similarity as this measure relies on SP vectors that contain no topics. It is indeed reasonable to expect that distributional measures, based on the distribution of topics in user profiles would work well on user-to-user similarity. The enrichment of problem topics using Linked Data-based measures, on the other hand, has already been shown to perform well in keyword suggestion scenarios [31] and it is reasonable to expect that an enrichment based on the meaning of topics would allow better mapping of the problem's conceptual space and reach users whose profiles have a more complete coverage of this space. 


\subsection{Evaluation 2}

In our second evaluation, we solicited ratings from 12 individual Twitter users, experts in the field of Semantic Web. Similar to the previous study, we provided them with the list of candidate co-solvers and asked them to select those that they would work with on a given problem, for which they were only partially competent. The same 3 problems were used by each rater, thereby resulting in 36 sets of rated suggestions. This time, in order to generate a more reusable gold standard, we asked the raters to evaluate all the possible user candidates we collected whose profiles contained at least one of the difference topics. Each set of suggestions for each problem-user pair contained 80-240 suggestions to evaluate. This was timeconsuming for raters but resulted in a gold standard that covered virtually all candidates that could be recommended by any variation of our approach. Such a gold standard allowed us to perform additional comparisons of methods, and especially focus on composite similarity measures that were not the subject of the first study.

Ranked candidate lists were generated using the following combinations of similarity functions and profile expansion methods:

- $\operatorname{PC}\left(\operatorname{Cosine}_{\mathrm{s}}, \mathrm{Cosine}_{\mathrm{dt}}, \mathrm{Cosine}_{\mathrm{t}}\right)$ : composite function that is a product of interest, social and the similarity with difference topics counted using cosine similarity.

- $\operatorname{PRF}\left(\operatorname{PC}\left(\operatorname{Cosine}_{\mathrm{s}}\right.\right.$, Cosine $\left.\left._{\mathrm{d} t}, \mathrm{Cosine}_{\mathrm{t}}\right)\right)$ : PRF problem profile expansion with composite similarity.

- $\quad$ PC $\left(\right.$ Cosine $_{\mathrm{s}}, \mathrm{HPSR}\left(\right.$ Cosine $\left.\left._{\mathrm{dt}}\right), \mathrm{Cosine}_{\mathrm{t}}\right)$ : HPSR expansion performed on difference topics prior to calculating the similarity with difference topics.

- PC $\left(\right.$ Cosine $_{\mathrm{s}}$, Cosine $_{\mathrm{dt}}, \mathrm{DMSR}\left(\right.$ Cosine $\left.\left._{\mathrm{t}}\right)\right)$ : DMSR expansion performed over the seed user profile prior to calculating interest similarity.

- $\operatorname{PC}\left(\right.$ Cosine $_{\mathrm{s}}, \operatorname{HPSR}\left(\right.$ Cosine $\left._{\mathrm{dt}}\right), \mathrm{DMSR}\left(\right.$ Cosine $\left.\left._{\mathrm{t}}\right)\right)$ : composite function in which HPSR is used to expand profile topics and DMSR to expand seed user topic profile prior to calculating the similarities.

In the above user study we described the results obtained when using hyProximity and Distributional profile expansion measures over the elementary similarity functions. For brevity, in this section, we omit these results from the second study and concentrate on the remaining permutations and their combinations using a composite similarity function - something that was not possible in the first study. We focus on composite measures as they allow us to gain a more complete insight in the impact of profile expansion on our multi-criteria recommendation task as a whole. As shown on Figure 3, according to the DCG measure for the first 10 ranked suggestions, the approaches with topic enrichment by either PRF, HPSR or DMSR consistently show better results than the basic approach, on all 3 problems. The overall values are on an expected level for the relevance scale used. HPSR performs slightly better then the other methods in most cases. However the mixed aggregate function (where HPSR is applied to the enrichment of problems and DMSR to the enrichment of user profiles) mostly gives lower results than the individual enrichment approaches. The cause of this might simply be that expanding both problem and seed user profiles induces too much of a difference with regards to the input data and might divert the co-solver search to a non-desired direction. The results shown on the Figure 4 represent the case when the Cosine similarity function is used. When the Weighted Overlap is used, results show negligible differences with the order of best alternatives unchanged, and are omitted for brevity reasons. 

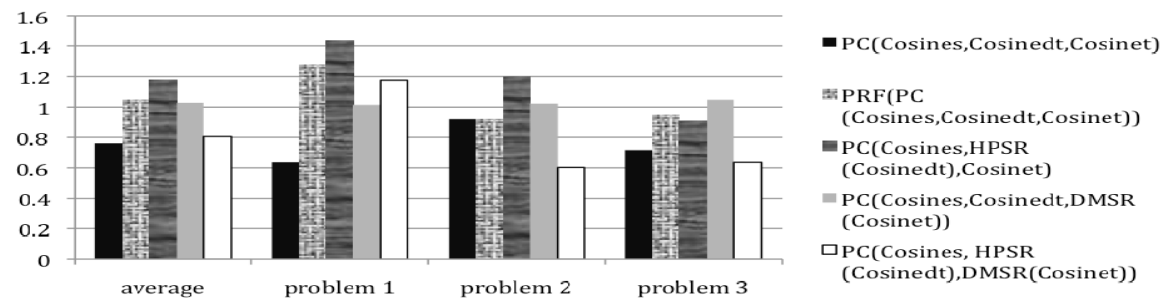

Fig. 3. Average DCG for all raters for different alternatives of composite similarity functions
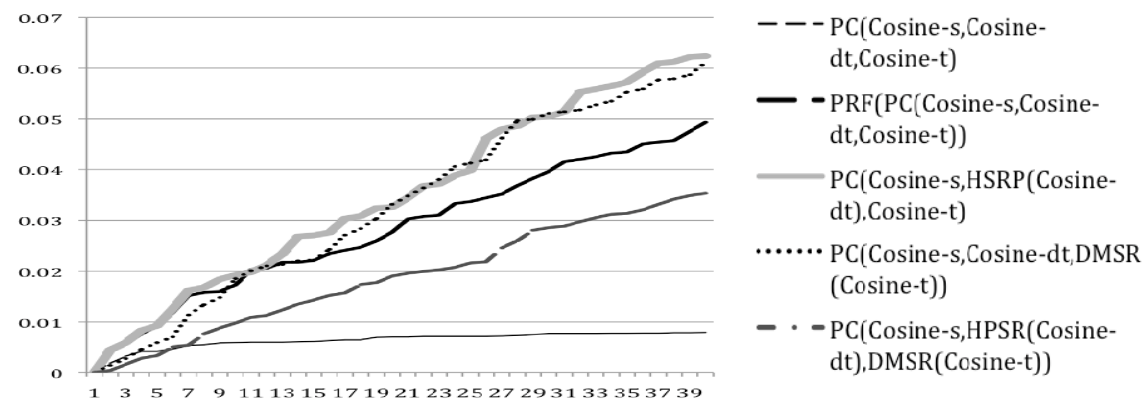

Fig. 4. AvePn of composite approaches (y axis), counted at different rank positions from 1-40 (x axis). Better approaches reach higher AveP at lower ranks.

Similar results are observed with the Average Precision used as the performance metric (FIgure 4). It shows that even on a larger set of best ranked candidates (40) the individual expansion methods dominate the mixed one. All the expansion methods also dominate the basic approach. The methods that gain higher values of AveP at lower numbers of rank positions are the ones that give more valuable suggestions at higher ranks and alow the user to discover valuable collaborators wile going through a lower number of suggestions. In this case, HSPR enrichment has slightly better results then the other methods. In order to give a better insight into the usefulness of rhe results generated with our aproach we provide an example of co-solver suggestions on our website ${ }^{12}$.

\section{Conclusions and Future Work}

In this paper we proposed an approach for suggesting co-solvers to a person engaged in solving a problem (industrial or research challenge) that has only partial competence and is looking for a complementary and compatible collaborator. Our work explored two central questions: how can we suggest appropriate co-solvers, which were potentially, previously unknown to the user? And: what information can be used to enrich initially available user and problem data to provide the best

${ }^{12}$ http: //research.hypios.com/?page_id=184 
suggestions? In addition to standard profiling techniques aimed at creating profiles with topic concepts referring to resources defined in the Linked Data Cloud, we proposed ways for expanding the profiles with additional relevant profiles in order to improve the final co-solver suggestions based on commonly used similarity methods. Through two user studies we have demonstrated that our Linked Data-based measure of semantic relatedness HPSR performs better then state of the art measures when applied with the expansion of problem profiles, while a commonly used measure based on the distribution of topics in user profiles performs better when it comes to expanding the seed user profile. When applied to composite similarity measures that reflect the social and interest similarity of candidate users (suggested co-solvers) with the seed user (user requiring help), as well as their complementarily with regards to the problem, all of the profile expansion methods outperform the simple approach, while HPSR used to expand problem profiles is slightly better than the others. Combined expansion of both problem and seed user's profiles does not outperform individual expansions. One of the future work directions will be to experiment with the graph-based measures of similarity, in addition to the vector measures presented in this paper.

Acknowledgments. The work of Milan Stankovic has been partially funded by ANRT under the grant number CIFRE N 789/2009. The work of Matthew Rowe was supported by the EU-FP7 projects WeGov (grant no. 248512) and Robust (grant no. 257859).

\section{References}

1. Jones, B.F., Wuchty, S., Uzzi, B.: Multi-university research teams: shifting impact, geography, and stratification in science. Science 322(5905), 1259-1262 (2008)

2. Hinds, P.J., Carley, K.M., Krackhardt, D., Wholey, D.: Choosing Work Group Members: Balancing Similarity, Competence, and Familiarity 1. Organizational Behavior and Human Decision Processes 81(2), 226-251 (2000)

3. Chesbrough, H.W.: Open Innovation: The New Imperative for Creating and Profiting from Technology. Harvard Business Press (2003)

4. Jeppesen, L.B., Lakhani, K.R.: Marginality and Problem Solving Effectiveness in Broadcast Research. Organization Science 20 (2009)

5. Spertus, E., Sahami, M., Buyukkokten, O.: Evaluating similarity measures. In: Proceedings of the 11th ACM SIGKDD International Conference on Knowledge Discovery in Data Mining, New York, USA (2005)

6. Luo, H., Niu, C., Shen, R., Ullrich, C.: A collaborative filtering framework based on both local user similarity and global user similarity. Machine Learning 72(3), 231-245 (2008)

7. Guy, I., Jacovi, M., Perer, A., Ronen, I., Uziel, E.: Same places, same things, same people?: mining user similarity on social media. In: Proceedings of the 2010 ACM Conference on Computer Supported Cooperative Work, pp. 41-50. ACM (2010)

8. Chen, J., Geyer, W., Dugan, C., Muller, M., Guy, I.: Make new friends, but keep the old: recommending people on social networking sites. In: Proceedings of the 27th International Conference on Human Factors in Computing Systems. ACM (2009)

9. Lakiotaki, K., Matsatsinis, N., Tsoukias, A.: Multicriteria User Modeling in Recommender Systems. IEEE Intelligent Systems 26(2), 64-76 (2011) 
10. Kazienko, P., Musial, K., Kajdanowicz, T.: Multidimensional Social Network in the Social Recommender System. IEEE Transactions on Systems, Man, and Cybernetics - Part A: Systems and Humans 41(4), 746-759 (2011)

11. Siersdorfer, S., Sizov, S.: Social recommender systems for web 2.0 folksonomies. In: Proceedings of the 20th ACM Conference on Hypertext and Hypermedia, HT 2009, p. 261. ACM Press, New York (2009)

12. Text Retrieval Conference Proceedings (1992-2010)

13. Zoltan, K., Johann, S.: Semantic analysis of microposts for efficient people to people interactions. In: 10th Roedunet International Conference, RoEduNet 2011, pp. 1-4, 23-25 (2011)

14. Ziaimatin, H.: DC Proposal: Capturing Knowledge Evolution and Expertise in Community-Driven Knowledge Curation Platforms. In: Aroyo, L., Welty, C., Alani, H., Taylor, J., Bernstein, A., Kagal, L., Noy, N., Blomqvist, E. (eds.) ISWC 2011, Part II. LNCS, vol. 7032, pp. 381-388. Springer, Heidelberg (2011)

15. Abel, F., Gao, Q., Houben, G.J., Tao, K.: Analyzing Temporal Dynamics in Twitter Profiles for Personalized Recommendations in the Social Web. In: Web Science Conference, Koblenz (2011)

16. Stan, J., Do, V.-H., Maret, P.: Semantic User Interaction Profiles for Better People Recommendation. In: Advances in Social Networks Analysis and Mining, ASONAM 2001 (2011); Rowe, M., Angeletou, S., Alani, H.: Predicting Discussions on the Social Semantic Web. In: Antoniou, G., Grobelnik, M., Simperl, E., Parsia, B., Plexousakis, D., De Leenheer, P., Pan, J. (eds.) ESWC 201. LNCS, vol. 6644, pp. 405-420. Springer, Heidelberg (2011)

17. Wagner, C.: Exploring the Wisdom of the Tweets: Towards Knowledge Acquisition from Social Awareness Streams. In: Aroyo, L., Antoniou, G., Hyvönen, E., ten Teije, A., Stuckenschmidt, H., Cabral, L., Tudorache, T. (eds.) ESWC 2010, Part II. LNCS, vol. 6089, pp. 493-497. Springer, Heidelberg (2010)

18. Burton-Jones, A., Storey, V.C., Sugumaran, V., Purao, S.: A Heuristic-Based Methodology for Semantic Augmentation of User Queries on the Web. In: Song, I.-Y., Liddle, S.W., Ling, T.-W., Scheuermann, P. (eds.) ER 2003. LNCS, vol. 2813, pp. 476489. Springer, Heidelberg (2003)

19. Ziegler, C.-N., Simon, K., Lausen, G.: Automatic Computation of Semantic Proximity Using Taxonomic Knowledge Categories and Subject Descriptors. In: Proceedings of the 15th ACM International Conference on Information and Knowledge Management, CIKM 2006, Arlington, Virginia, USA, pp. 465-474. ACM, New York (2006)

20. Strube, M., Ponzetto, S.P.: WikiRelate! Computing semantic relatedness using Wikipedia. In: Proceedings of the National Conference on Artificial Intelligence, vol. 21, p. 1419. AAAI Press, MIT Press, Menlo Park, Cambridge (1996, 2006)

21. Resnik, P.: Using Information Content to Evaluate Semantic Similarity in a Taxonomy (1995)

22. Matos, S., Arrais, J.P., Maia-Rodrigues, J., Oliveira, J.L.: Concept-based query expansion for retrieving gene related publications from Medline. BMC Bioinformatics 11, 212 (2010)

23. Cilibrasi, R.L., Vitanyi, P.M.B.: The Google Similarity Distance. IEEE Transactions on Knowledge and Data Engineering 19(3), 370-383 (2007), doi:10.1109/TKDE.2007.48

24. Macdonald, C., Ounis, I.: Expertise drift and query expansion in expert search. In: Proceedings of the Sixteenth ACM Conference on Information and Knowledge Management - CIKM 2007, vol. 341. ACM Press, New York (2007) 
25. Serdyukov, P., Chernov, S., Nejdl, W.: Enhancing Expert Search Through Query Modeling. In: Amati, G., Carpineto, C., Romano, G. (eds.) ECIR 2007. LNCS, vol. 4425, pp. 737-740. Springer, Heidelberg (2007)

26. Rizzo, G., Troncy, R.: NERD: Evaluating Named Entity Recognition Tools in the Web of Data. In: Proceedings of the 11th Interational Semantic Web Conference 2011, Bonn, Germany (2011)

27. Li, Q., Zheng, Y., Xie, X., Chen, Y., Liu, W., Ma, W.-Y.: Mining user similarity based on location history. In: Proceedings of the 16th ACM SIGSPATIAL International Conference on Advances in Geographic Information Systems - GIS 2008, p. 1. ACM Press, New York (2008)

28. Balog, K., de Rijke, M.: Finding similar experts. In: Proceedings of the 30th Annual International ACM SIGIR Conference on Research and Development in Information Retrieval, SIGIR 2007, p. 821. ACM Press, New York (2007), doi:10.1145/1277741.1277926

29. Viswanathan, K.K., Finin, T.: Text Based Similarity Metrics and Deltas for Semantic Web Graphs. In: Patel-Schneider, P.F., Pan, Y., Hitzler, P., Mika, P., Zhang, L., Pan, J.Z., Horrocks, I., et al. (eds.) Proceedings of the 9th International Semantic Web Conferene ISWC 2010, Shanghai, China (2010)

30. Stankovic, M., Breitfuss, W., Laublet, P.: Linked-Data Based Suggestion of Relevant Topics. In: Proceedings of I-SEMANTICS Conference 2011, Gratz, Austria, September 79 (2011)

31. Letierce, J., Passant, A., Decker, S., Breslin, J.: Understanding how Twitter is used to spread scientific messages. In: Proceedings of the WebSci 2010, Raleigh, NC, US (2010)

32. Fleiss, J.: Statistical Methods for Rates and Proportions. Wiley-Interscience (1981) 